\title{
All Three Endogenous Quinone Species of Escherichia coli Are Involved in Controlling the Activity of the Aerobic/Anaerobic Response Regulator ArcA
}

\author{
Johan W. A. van Beilen and Klaas J. Hellingwerf*
}

Department of Molecular Microbial Physiology, Swammerdam Institute for Life Sciences, University of Amsterdam, Amsterdam, Netherlands

\section{OPEN ACCESS}

Edited by:

Lars E. P. Dietrich,

Columbia University, USA

Reviewed by:

Katja Bettenbrock,

Max Planck Society, Germany

Davide Zannoni,

University of Bologna, Italy

TIm Rasmussen,

University of Aberdeen, UK

*Correspondence:

Klaas J. Hellingwerf

k.j.hellingwerf@uva.n

Specialty section:

This article was submitted to Microbial Physiology and Metabolism,

a section of the journal

Frontiers in Microbiology

Received: 08 May 2016

Accepted: 15 August 2016 Published: 07 September 2016

Citation:

van Beilen JWA and Hellingwerf $K J$ (2016) All Three Endogenous Quinone

Species of Escherichia coli Are

Involved in Controlling the Activity of the Aerobic/Anaerobic Response

Regulator ArcA.

Front. Microbiol. 7:1339.

doi: 10.3389/fmicb.2016.01339
The enteron Escherichia coli is equipped with a branched electron transfer chain that mediates chemiosmotic electron transfer, that drives ATP synthesis. The components of this electron transfer chain couple the oxidation of available electron donors from cellular metabolism (e.g., NADH, succinate, lactate, formate, etc.) to the reduction of electron acceptors like oxygen, nitrate, fumarate, di-methyl-sulfoxide, etc. Three different quinones, i.e., ubiquinone, demethyl-menaquinone and menaquinone, couple the transfer of electrons between the dehydrogenases and reductases/oxidases that constitute this electron transfer chain, whereas, the two-component regulation system ArcB/A regulates gene expression, to allow the organism to adapt itself to the ambient conditions of available electron donors and acceptors. Here, we report that $E$. coli can grow and adjust well to transitions in the availability of oxygen, with any of the three quinones as its single quinone. In all three 'single-quinone' E. coli strains transitions in the activity of ArcB are observed, as evidenced by changes in the level of phosphorylation of the response regulator ArcA, upon depletion/readmission of oxygen. These results lead us to conclude that all quinol species of $E$. coli can reduce (i.e., activate) the sensor ArcB and all three quinones oxidize (i.e., de-activate) it. These results also confirm our earlier conclusion that demethyl-menaquinone can function in aerobic respiration.

Keywords: ubiquinone, menaquinol, naphtoquinones, phos-tag electrophoresis, single-quinone producing mutants, ubiE

\section{INTRODUCTION}

Redox- and phosphoryl-transfer reactions, with coupled chemi-osmotic components, form the core of cellular energetics (Hellingwerf and Konings, 1985). The stepwise release of redox potential in a central electron-transfer system ('chain') is fundamental to the vast majority of living cells (Nicholls and Ferguson, 2013). On the other hand (oxygen) radical formation, catalyzed as a side reaction by the same system, is one of the most important causes of damage to the cell (Messner and Imlay, 1999). Therefore, cellular redox reactions need to be tightly regulated. Escherichia coli is a facultative (an)aerobic bacterium and it therefore needs to be able to adapt its metabolism, and respiration, to varying oxygen concentrations. Under aerobic conditions, oxygen acts as the 
preferred terminal electron acceptor (Madigan and Martinko, 2014). When the supply of oxygen becomes limiting, chemically reduced metabolites and electron carriers in their reduced form may accumulate. This ultimately can inhibit growth by oxidative damage (Messner and Imlay, 1999). Under fully anaerobic conditions, alternative terminal electron acceptors, such as nitrate and dimethylsulfoxide (DMSO) may be used to substitute for the role of $\mathrm{O}_{2}$ (Table 1), and if all of these are absent, the cell may switch to fermentation as a last resort.

Accordingly, under both aerobic and anaerobic conditions, E. coli manages to proliferate. Anaerobically, a variety of terminal electron acceptors can be used to re-oxidize the NADH pool, generated by the upstream part of central metabolism. The midpoint potential, however, of these alternative electron acceptors is less positive than of the oxygen/water pair (Table $\mathbf{1}$ ). When requiring alternative electron acceptors, the cell needs to change gears with respect to the respiratory chain and use fewer or smaller steps of redox potential to channel electrons from $\mathrm{NADH}$ to the terminal electron acceptor, for the generation of a proton motive force and coupled ATP synthesis, be it that also the magnitude of the proton gradient itself may decrease (Hellingwerf et al., 1981; Tran and Unden, 1998).

The respiratory chain of $E$. coli contains considerable redundancy with respect to the components required for each step of electron transfer from NADH to the terminal electron acceptor (Unden and Bongaerts, 1997). This presumably enables the cell to adjust the metabolic yield of this electron transfer to the needs of the cell, when operating in different metabolic modes. Wild type E. coli (including strain MG1655 used in this study) contains several dehydrogenases that oxidize cytoplasmic electron donors, like $\mathrm{NADH}$, and donate electrons to the quinones embedded in the membrane (or quinols when they are present in their reduced form (Sharma et al., 2012). Under aerobic conditions the quinols are then oxidized by any of three terminal oxidases, which transfer electrons to the terminal electron acceptor with slight preference (Sharma et al., 2012). This electron transfer chain has been studied extensively during the past decades, both by others and by ourselves (Van Der Plas et al., 1983; Visser et al., 1984; van Schie et al., 1985; Poole and Cook, 2000; Bekker et al., 2009; Sharma et al., 2012; Steinsiek et al., 2014).

\begin{tabular}{|c|c|c|}
\hline Chemical moiety & $\begin{array}{c}\text { Midpoint } \\
\text { potential }(\mathrm{mV})\end{array}$ & Reference \\
\hline $\mathrm{NAD} / \mathrm{NADH}_{2}$ & -320 & Unden and Bongaerts, 1997 \\
\hline $\mathrm{MK} / \mathrm{MKH}_{2}$ & -72 & Unden and Bongaerts, 1997 \\
\hline ArcB & -41 & Alvarez et al., 2013 \\
\hline Fumarate/succinate & 30 & Unden and Bongaerts, 1997 \\
\hline $\mathrm{DMK} / \mathrm{DMKH}_{2}$ & 36 & Unden and Bongaerts, 1997 \\
\hline UQ/UQH ${ }_{2}$ & 110 & Unden and Bongaerts, 1997 \\
\hline Dimethylsulfoxide /DMS* & 160 & Unden and Bongaerts, 1997 \\
\hline $\mathrm{O}_{2} / \mathrm{H}_{2} \mathrm{O}$ & 820 & Unden and Bongaerts, 1997 \\
\hline
\end{tabular}

*Dimethyl sulfide.
In E. coli, there are two primary $\mathrm{NADH}$ dehydrogenases (NDH-I and NDH-II) that are part of the standard respiratory chain and that may transfer electrons to any of the three endogenous quinones. In addition, formate dehydrogenase, lactate dehydrogenase, and succinate dehydrogenase may also donate electrons to the quinone pool, thereby oxidizing their respective substrate. The quinone pool of $E$. coli consists of three quinone types, the benzoquinone ubiquinone (UQ) and the naphtoquinones demethylmenaquinone (DMK) and menaquinone (MK); for reviews, see (Unden and Bongaerts, 1997; Sharma et al., 2012), with on average a prenyl sidechain length of 8 (Meganathan, 2001b). Ubiquinone is primarily used during aerobic respiration and nitrite respiration (be it, together with MK; Sharma et al., 2012). Menaquinone is the primary quinone under anaerobic conditions. It is amongst others specifically required for formate dehydrogenase activity and for DMSO (dimethylsulfoxide) reduction (Wissenbach et al., 1990). In agreement with this it has been observed that the total level (i.e., oxidized plus reduced) of the ubiquinones increases, and the total level of the menaquinones decreases, when aerobiosis is gradually increased from 0 to $100 \%$ (Bekker et al., 2010). DMK has a rather broad specificity regarding its ability to react with dehydrogenases and reductases/oxidases (Unden and Bongaerts, 1997; Sharma et al., 2012). The three terminal oxidases of $E$. coli, cytochrome bo oxidase, cytochrome $b d$ I oxidase and cytochrome $b d$ II oxidase (Bekker et al., 2009), are somewhat specific in terms of the quinol electron donor that they prefer, one of the determining factors being their mutual redox midpoint potential (Unden and Bongaerts, 1997), but all three can oxidize both ubiquinol and demethylmenaquinol (Sharma et al., 2012). Other terminal electron acceptors use dedicated enzymes such as nitrate (NarGHI), fumarate (FrdABCD), DMSO (DmsABC), TMAO (trimethylamine $\mathrm{N}$-oxide; TorCDA), and nitrite (NrfABCD), each with their own quinone specificity (Unden and Bongaerts, 1997). A final complicating factor in understanding specificity in electron transfer reactions in the electron transfer chain of $E$. coli are the reactions between reduced and oxidized species of the different quinones. Although very little solid data on this aspect is available, our previous studies indicated that this aspect cannot be ignored (Bekker et al., 2010).

For any E. coli cell living in the ambient environment, the transition from anaerobic to aerobic conditions and vice versa is one of the most thoroughgoing transitions. Going through this transition requires alteration of the functional activity of a large number of metabolic- and respiratory pathways, to the extent that the transcription of some 200 genes has to be adjusted in this transition (Salmon et al., 2005). A large part of this transcriptional regulation is carried out by the Anoxic Redox Control (Arc) two-component system, that functions complementary to three additional systems: FNR, SoxRS, and OxyR (for a review see Iuchi and Weiner, 1996). The Arc twocomponent system consists of the classical response regulator ArcA, and the redox-sensitive, membrane-embedded kinase, ArcB. ArcA can both be phosphorylated (leading to formation of the transcriptionally active state) and dephosphorylated (its inactive state) by ArcB. 
The signal-induced switching of the activity of ArcB, between its kinase- and phosphatase function, is the subject of this study. After an early consensus that it could not be molecular oxygen that is the signal for ArcB, Georgellis et al. (2001) convincingly showed that in vitro, $\mathrm{UQ}_{0}$ inhibits the autophosphorylation of $\mathrm{ArcB}$ - and by inference its kinase activity - and $\mathrm{UQ}_{0} \mathrm{H}_{2}$ actives it. With Alexeeva et al. (2002) we introduced a system that allows titration of the rate of electron transfer to oxygen in chemostat-grown cells of E. coli - the aerobiosis system. If it would be only the redox state of the ubiquinone pool that would regulate the activity of the $\mathrm{ArcB}$ sensor one would predict that in this aerobiosis system a sigmoidal relation would be obtained between $\mathrm{ArcB}$ activity and the degree of aerobiosis (reflecting the gradual transition of predominance of ubiquinol/ubiquinone). We did observe, however, a relation that was more complex (Alexeeva et al., 2002; Bekker et al., 2010), and interpreted this result as a consequence of the fact that not only ubiquinol but also (dimethyl)menaquinol would be able to reduce, and hence activate, $\mathrm{ArcB}$. Conversely, other authors have found different relations between aerobiosis and $\mathrm{ArcB}$ activity (e.g., Rolfe et al., 2011; Alvarez et al., 2013; Steinsiek et al., 2014)

Although, meanwhile we have provided more evidence supporting our interpretation (Sharma et al., 2013), Alvarez et al. (2013) have published an updated model for the regulation of ArcB activity by the quinone pool of E. coli, based on an in vivo determination of the redox midpoint potential of the redox-sensitive active site cysteine(s) residue of $\mathrm{ArcB}$. Using the system of cysteine/cystine redox buffers a value of $-41 \mathrm{mV}$ was obtained for one (or both) of the critical cysteines that have been assigned to this role. They went then on to argue that it would be logical to assume that ubiquinone (with a midpoint potential of $+100 \mathrm{mV}$ ) can only inactivate ArcB (and by inference: ubiquinol cannot activate it), although we have clearly demonstrated (amongst others through the use of socalled UQ-only mutants) that both ubiquinol and menaquinol can both activate ArcB in E. coli (Bekker et al., 2010; Sharma et al., 2012). Nevertheless, Alvarez et al. (2013) postulate their 'yin and yang' model for ArcB regulation: exclusive activation of ArcB by menaquinol (midpoint potential: $-74 \mathrm{mV}$ ) and exclusive inactivation by ubiquinone.

The laws of thermodynamics, however, hold that whether or not two redox active molecules will react - besides chemical specificity - is not so much dictated by their redox midpoint potential, but rather by the actual redox potential of the two couples involved (Berg et al., 2002). Therefore, there is no compelling a priori reason to assume that high concentrations of ubiquinol would not be able to activate ArcB, nor high concentrations of menaquinone to de-activate it, particularly if activation of a subset of $\mathrm{ArcB}$ molecules would suffice to increase the net phosphorylation level of ArcA. Therefore, we have constructed three mutants of $E$. coli that contain a single quinone type only, and studied ArcB regulation in these strains. Consistent with our previous observations, here we report that all three 'single-quinone' mutants display the ArcB activation/deactivation cycle.
For the construction of the menaquinone-only mutant we cloned the ubiE homolog menH from Bacillus subtilis in E. coli. The resulting mutant strain turned out to convert essentially all its demethylmenaquinone/ol into menaquinone/ol.

\section{MATERIALS AND METHODS}

\section{Strains Used in this Investigation}

All strains used in this study are derived from the E. coli K12 wild type strain MG1655 (Table 2). This strain contains all three biologically active quinones that are known to be present in E. coli. The deletion mutants AV34 and AV36, containing only UQ and only DMK, respectively, were obtained from our own strain collection (Sharma et al., 2013). The DMKonly and MK-only strains were constructed using the Gene Doctoring system (Lee et al., 2009). Plasmids were designed and constructed based on $\mathrm{pDOC}-\mathrm{K}$, to delete $u b i C A$ or introduce $u b i E$ or $m e n H_{B s u}$ and combinations of these, using a spectinomycin resistance cassette (-S) from pDG1661 (Guérout-Fleury et al., $1996)$ instead of the kanamycin resistance cassette (-K). The $u b i E$ gene or its homolog menH from B. subtilis were genomically integrated in the yoe $G$ locus, which we assumed to be a neutral site, by using pDOC-K-yoeG-ParaMenH [note that yoeG is annotated as the defective integrase of the CP4-44 prophage (Wang et al., 2010)]. For all plasmids, 250 bp of the $3^{\prime}$ and $5^{\prime}$ ends of the target locus were cloned into the pDOC vector, to allow homologous replacement with the resistance cassette (and additional genes when present). The arabinose inducible promotor used $\left(\mathrm{P}_{\text {ara }}\right)$ was PCR amplified from pACBSCE and fused to the downstream gene with an overlap-extension PCR. For overexpression of $m e n H_{B s u}, 0.1 \%$ arabinose $(\mathrm{w} / \mathrm{v})$ was used.

It is important to note that selection of MK-only E. coli mutants was successful only (in our hands) when LB-agar plates, containing $20 \mathrm{mM}$ glucose and $0.2 \mathrm{mM} \mathrm{UQ}_{0}$, were used under strict anaerobic conditions, in an anaerobic jar flushed with $\mathrm{N}_{2}$ and containing an AnaeroGen pack (Oxoid, Thermo Scientific) to eliminate remaining traces of $\mathrm{O}_{2}$. When cultured under (micro)aerobic conditions for longer periods of time, multiple

TABLE 2 | Strain and plasmids used in this study.

\begin{tabular}{lll}
\hline Name & Genotype & Reference \\
\hline E. coli MG1655 & K-12 wild type & Lab stock \\
E. coli AV34 & $\Delta$ menA::kan & Sharma et al., 2013 \\
E. coli AV36 & $\Delta$ ubiE::kan & Sharma et al., 2013 \\
E. coli DMK-only & $\Delta$ ubiE::kan $\Delta$ ubiCA::Spc & This study \\
E. coli MK-only & $\Delta$ ubiCA::Spc $\Delta y o e G::$ Kan & This study \\
& $P_{\text {ara-menH }}$ Bsu & \\
pACBSCE & [see reference] & Lee et al., 2009 \\
pDOC-K & [see reference] & Lee et al., 2009 \\
pDOC-K-yoeG- & See text, Supplementary & This study \\
ParaMenH & Figure S1A & \\
pDOC-S-ubiCA & See text, Supplementary & This study \\
& Figure S1B &
\end{tabular}


revertant mutants could be picked up, containing DMK only or $\mathrm{DMK}+\mathrm{MK}$, which in liquid cultures outcompete the MK-only strain.

\section{Growth of the E. coli Strains in Batch Culture}

All strains were inoculated from single colonies and pre-cultured in LB medium containing $20 \mathrm{mM}$ glucose and $50 \mathrm{mM}$ DMSO (dimethylsulfoxide) at $37^{\circ} \mathrm{C}$ under continuous agitation with anaerobic conditions in a jar flushed with nitrogen gas. This culture was used as a $0.1 \%$ inoculum for $25 \mathrm{ml}$ cell cultures which were grown as anaerobic batch culture at $37^{\circ} \mathrm{C}$ using Evans' salt medium with nitrilo-acetic acid $(2 \mathrm{mM})$ and sodium phosphate buffer (100 mM, pH 7) to increase buffering capacity (Evans et al., 1970; Sharma et al., 2013). Glucose (20 mM) was used as carbon source and $50 \mathrm{mM}$ DMSO was used as terminal electron acceptor. For anaerobic transition experiments, the contents of these were then transferred to a batch fermenter containing $500 \mathrm{ml}$ of Evans' medium containing $20 \mathrm{mM}$ glucose, no extra DMSO and continuous nitrogen gas sparging at a flow rate of $50-80 \mathrm{ml} / \mathrm{min}$ to maintain anaerobic conditions. These anaerobic cultures, were used for the anaerobic/aerobic transition experiments, keeping all the other conditions the same. All strains were assessed in at least three biologically independent replicates.

\section{Quinone Extraction and Analysis}

The extraction and analysis of quinones was carried out essentially as described by Bekker et al. (2007). Briefly, at each time point a $2 \mathrm{ml}$ sample was taken in $6 \mathrm{ml}$ of a $1: 1(\mathrm{v} / \mathrm{v})$ mixture of ice-cold methanol and petroleum ether (evaporation temperature $40-60^{\circ} \mathrm{C}$ ). The mixture then was vortexed for $1 \mathrm{~min}$ and centrifuged at 3,000 $\mathrm{rpm}$ for $1 \mathrm{~min}$. Then the upper petroleum ether phase was transferred to a glass tube under a nitrogen atmosphere and containing $80 \mu \mathrm{l}$ 1-hexanol. After evaporation of the petroleum ether (20-30 min), the 1-hexanol was transferred to a glass high-performance liquid chromatography (HPLC) vial and stored at $-20^{\circ} \mathrm{C}$ until analysis within $48 \mathrm{~h}$.

The samples were fractionated with HPLC using a reversed-phase Lichrosorb (Chrompack, Bergen op Zoom, The Netherlands) RP10 C18 column (size, $4.6 \mathrm{~mm}$; internal diameter, $250 \mathrm{~mm}$ ). The column was equilibrated with pure methanol as the mobile phase at the flow rate of $2 \mathrm{ml} / \mathrm{min}$. Detection of the quinones was performed using a UV/Vis absorption detector at $290 \mathrm{~nm}$ for ubiquinone (UQ) and at $248 \mathrm{~nm}$ for naphtoquinones (DMK and MK). All reduced quinone species were also detected with an Agilent 1200 series fluorescence detector, coupled in series with a UV/Vis detector, using 238 and $375 \mathrm{~nm}$ as the excitation- and emission wavelength, respectively, with the photomultiplier gain set to 12 . The amount of each quinone species was calculated from the relevant area under the peak.

Standards of all (oxidized) quinone species were prepared by isolating relevant fractions from the HPLC and re-extracting these as described above. Concentrated and purified quinones were analyzed on a UV/Vis spectrophotometer and based on their respective extinction coefficient (White, 1965; Holländer, 1976), their concentrations were determined. The $\mathrm{UQ}_{8}$ used in some experiments to assist the respiratory chain of the MK only strain were obtained as described above, from cultures of E. coli AV34.

\section{Measurement of ArcA Phosphorylation with Phos-tag Electrophoresis}

Relative ArcA phosphorylation levels were measured with Phos-tag ${ }^{\mathrm{TM}}$-acrylamide gel electrophoresis and Western immunoblotting as described by Rolfe et al. (2011). The purified ArcA and antibodies were obtained as described (Bekker et al., 2010). In vitro phosphorylation was achieved by incubation of ArcA in $30 \mathrm{mM}$ HEPES pH 7.5, $10 \mathrm{mM} \mathrm{MgCl}_{2}, 25 \mathrm{mM}$ acetyl phosphate and $10 \%$ glycerol. All samples were taken from at least three biologically independent replicates, sampled in technical duplicates.

\section{Metabolite Analysis}

Samples from each time point were processed for HPLC analysis essentially as described before (Sharma et al., 2012); a $1 \mathrm{ml}$ sample was mixed with $100 \mu \mathrm{l} 35 \%$ perchloric acid and subsequently $55 \mu 17 \mathrm{M} \mathrm{KOH}$ was added. Filtered supernatants were analyzed for glucose consumption and fermentation products. Glucose, pyruvate, lactate, formate, acetate, succinate, and ethanol contents were determined by HPLC (LKB and Pharmacia, Oregon City, OR, USA) using a REZEX organic acid analysis column (Phenomenex, Torrance, CA, USA) at $45^{\circ} \mathrm{C}$, with $7.2 \mathrm{~mm} \mathrm{H}_{2} \mathrm{SO}_{4}$ as the eluent, using an RI 1530 refractive index detector (Jasco, Easton, MD, USA) and AZUR chromatography software (St. Martin D'Heres, France) for data analysis.

\section{RESULTS}

\section{Construction of Three 'Single Quinone' E. coli Strains}

To characterize the role of the various quinones of E. coli in the regulation of the activity of two-component kinase/phosphatase $A r c B$, we set out to construct a set of E. coli strains that have only one single quinone species. In E. coli, three dominant quinone types are found that are active in facilitating electron transfer in the respiratory chain (Sharma et al., 2012). These are the benzoquinone ubiquinone (UQ) and two naphtoquinones, demethylmenaquinone (DMK) and menaquinone (MK; see also Figure 1). From a batch culture of wild type E. coli MG1655, both the oxidized and reduced form of all three types of quinone can be identified, as is shown in Figure 2A. We note, however, that without specific precautions the oxidized/reduced (ox/red) ratio observed in such samples for the menaquinones does not accurately reflect their in vivo ox/red ratio because of the rapid spontaneous auto-oxidation, in contrast to the observations made with ubiquinone (Bekker et al., 2010; Sharma et al., 2013). Furthermore, for optimal quantification of these six species three different detector settings are required, for absorption at 248 and at $290 \mathrm{~nm}$ and for fluorescence emission, respectively. 


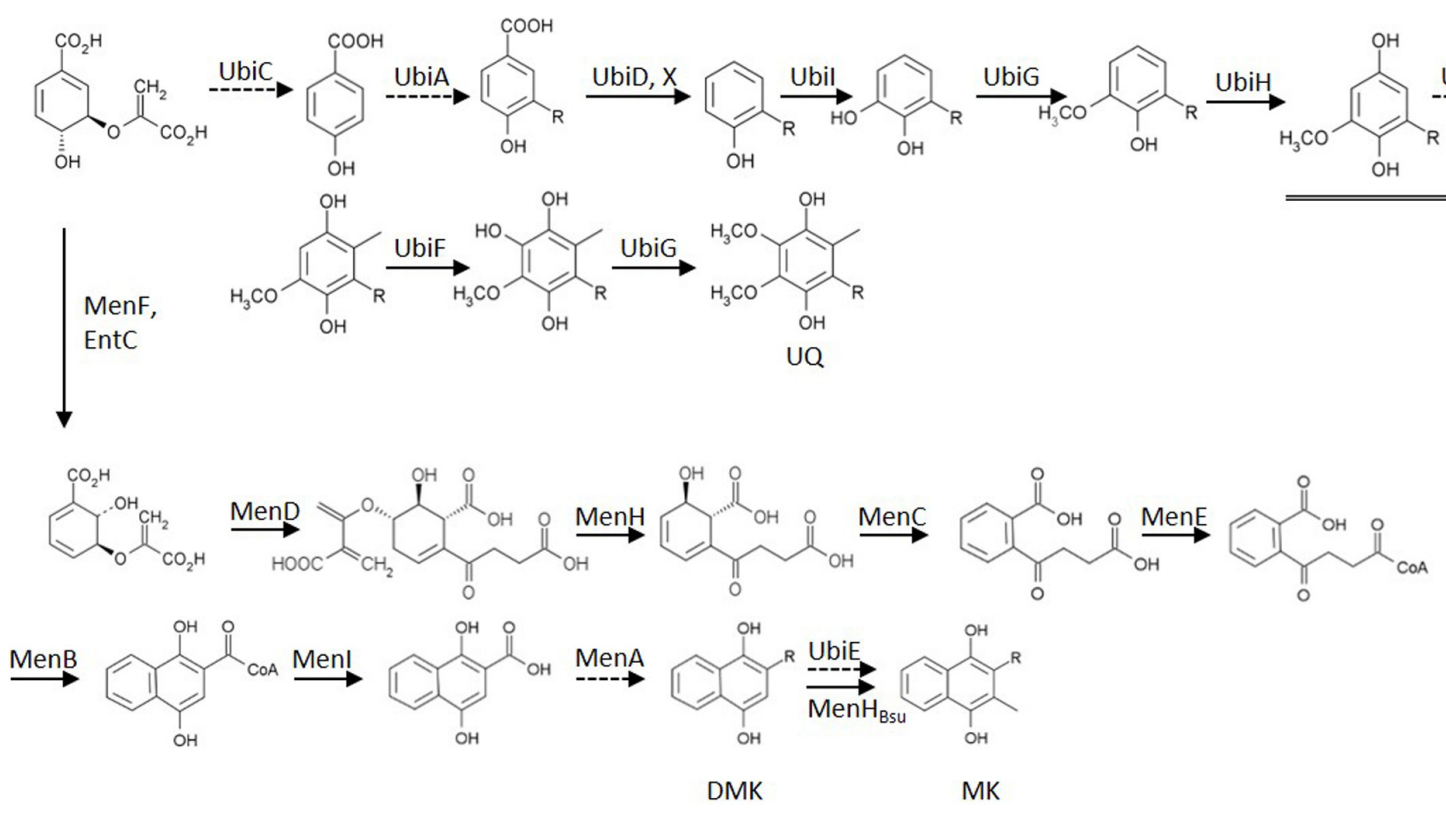

FIGURE 1 | Biosynthesis routes of the quinones of Escherichia coli, starting from chorismate. Dashed arrows indicate enzymes deleted in mutants used in this study. The double arrow symbolizes the action of the introduced heterologous MenHBsu. The underlined intermediate C1-demethyl-C6-demethoxy-Q8 (DDMQ8) may accumulate in a ubiE mutant strain (for references: see text). Established bio-active quinones are indicated via their abbreviation. UQ, ubiquinone; DMK, demethylmenaquinone; MK, menaquinone; R, isoprenoid sidechain.

Strains were available from previous studies that contain as their sole quinone either ubiquinone (i.e., AV34) or demethylmenaquinone (AV36; Sharma et al., 2013). DMK is the direct precursor of $\mathrm{MK}$ and its methylation is catalyzed by UbiE (Lee et al., 1997). A knock-out of only ubiE, however, would lead to accumulation of C1-demethyl-C6-demethoxy-Q8 (DDMQ; see Figure 1). DDMQ most probably is redox-active (our unpublished results and Aussel et al., 2014) and might substitute for UQ, together with DMK in electron transfer reactions in the electron transfer chain. Therefore a $\Delta u b i E$ strain would not be suitable for proper analysis of the role of DMK. Instead, it is important to use a $\triangle u b i E \triangle u b i C A$ double mutant for such experiments. Such a mutant strain was constructed by knocking out $u b i C A$ in AV36 $(\triangle u b i E)$. This was achieved via the use of plasmid pDOC-S-ubiCA and the resulting strain is referred to as the 'DMK only' strain in the remainder of this communication.

An E. coli strain with MK as its sole quinone cannot be created by a gene knock-out only. The alternative approach that we initially selected, i.e., overexpression of $u b i E$ in a $\triangle u b i C A$ mutant strain (i.e., a strain that does not contain ubiquinone) did not result in complete conversion of DMK to MK (our unpublished results). The Gram-positive soil-dwelling bacterium B. subtilis uses $\mathrm{MK}$ as the sole quinone in its respiratory chain. Because the biosynthesis pathway of MK in B. subtilis is very similar to that of E. coli, we decided to overexpress the UbiE homolog MenH from B. subtilis in E. coli MG1655 and in its $\triangle$ ubiCA derivative strain.

As can be seen in Figure 2B, induced expression of a genomeintegrated copy of menH completely eliminated the presence of DMK in the mutant strain. Subsequent long term cultivation of this MK-only strain under aerobic conditions resulted in the emergence of revertant strains that readily out-competed the original mutants via a mutation that altered expression of one or both of the methyltransferases (i.e., giving rise to a DMK plus MK- or even DMK-only phenotype; our unpublished results). However, anaerobic cultivation with DMSO as the terminal electron acceptor resulted in dense cultures within $48 \mathrm{~h}$ with a stable, MK-only phenotype (Figure 2B). Figure 2B also confirms the phenotype of the other two single quinone strains. The components visible in the MK only strain eluting at $22 \mathrm{~min}$, and the fluorescent component in MG1655 eluting at $17 \mathrm{~min}$. have not been further identified.

\section{The Phosphorylation State of ArcA}

We next carried out a series of (an)aerobic transition experiments with the wild type strain and the three single quinone strains (Figure 3). The strains were pre-cultured under anaerobic conditions in Evans' medium, with glucose as the carbon and energy source, and subsequently transferred to shake-flasks and flushed with $\mathrm{N}_{2}$. After a few hours of growth, the cultures were switched from sparging with $\mathrm{N}_{2}$ to sparging with compressed air $(60 \mathrm{~min})$, and then back to $\mathrm{N}_{2}(60 \mathrm{~min})$, during which samples were taken. It can be seen that the growth rate of all three single quinone strains is reduced compared to that of the wild type strain. Cells sampled from these cultures were then assessed for the phosphorylation state of ArcA (Figure 4) and the redox state of their (ubi)quinone pool (Supplementary Figure S2).

The anaerobic- to aerobic shift assay with MG1655 shows that both kinase as well as phosphatase activity of ArcB can 


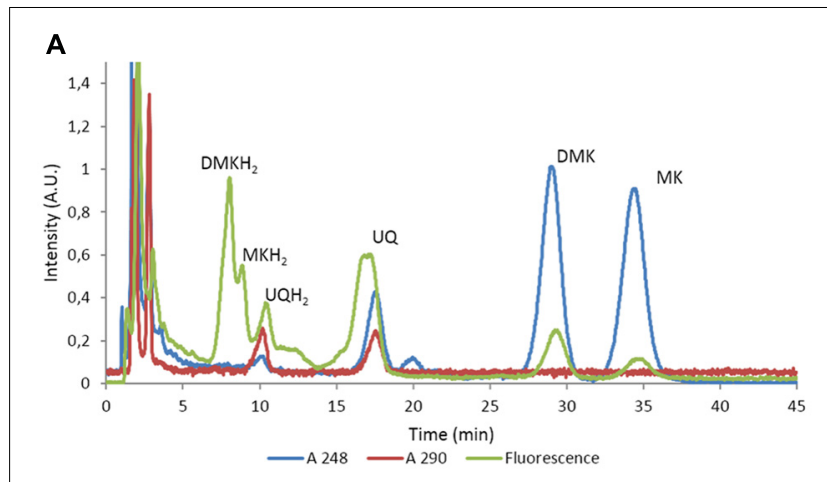

B

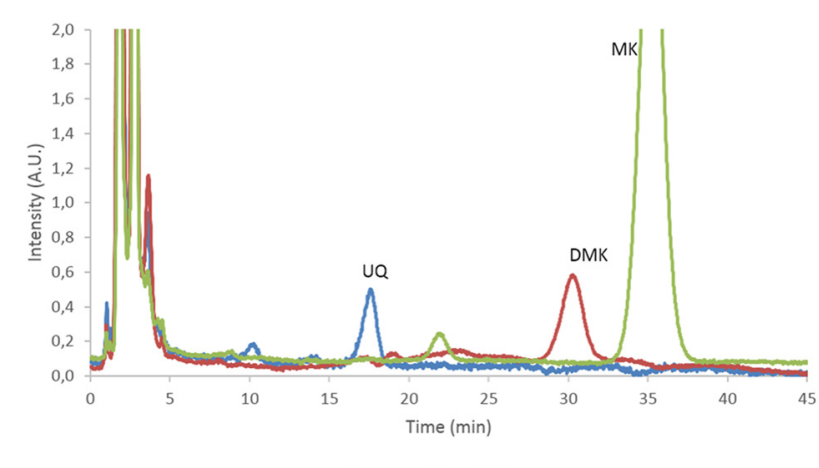

FIGURE 2 | Separation and quantification of the main quinone types of E. coli. (A) High-performance liquid chromatography (HPLC) trace of E. coli MG1655 with the eluate analyzed with $A_{248}, A_{290}$ and fluorescence. The identified quinones are indicated. $U Q$, ubiquinone; DMK, demethylmenaquinone; MK, menaquinone. Note that UQ is not fluorescent at these wavelengths and that the peak in the fluorescence channel at approximately 17 min. does not correspond to UQ. (B) HPLC traces recorded at $248 \mathrm{~nm}$ of E. coli strains AV34, DMK-only (AV36), MK-only (i.e., E. coli $\triangle$ ubiCA complemented with menH from Bacillus subtilis. $\cup Q$, ubiquinone; DMK, demethylmenaquinone; MK, menaquinone.

be observed (Figure 4A). However, during the first $40 \mathrm{~min}$ of the experiment generally a lower level of $A r c A \sim P$ was observed than the level shortly after switching back to anoxic conditions. This is consistent with earlier experiments which showed highest $\mathrm{UQH}_{2}$ /UQ ratios immediately upon anaerobiosis (Bekker et al., 2007). The UQ-only strain AV34 had a far stronger ArcA $\sim \mathrm{P}$ signal under anoxic conditions and this signal quickly disappeared when the cells were exposed to oxygen (Figure 4B). This shows that ubiquinone is able to facilitate the transition of ArcB's activity in both directions, i.e., $\mathrm{UQH}_{2}$ can activate and UQ can inactivate $\mathrm{ArcB}$, contrasting the claim that $\mathrm{UQH}_{2}$ cannot activate this kinase because of presumed incompatibility of the respective midpoint potential values.

In the DMK-only strain, ArcA $\sim \mathrm{P}$ could also be detected. However, the level of phosphorylated ArcA in this strain is lower than in the wild type and in AV34 (the UQ-only strain), but here too, a clear trend during the anarobiosis/aerobiosis transition could be observed (Figure 4C). This is possibly due

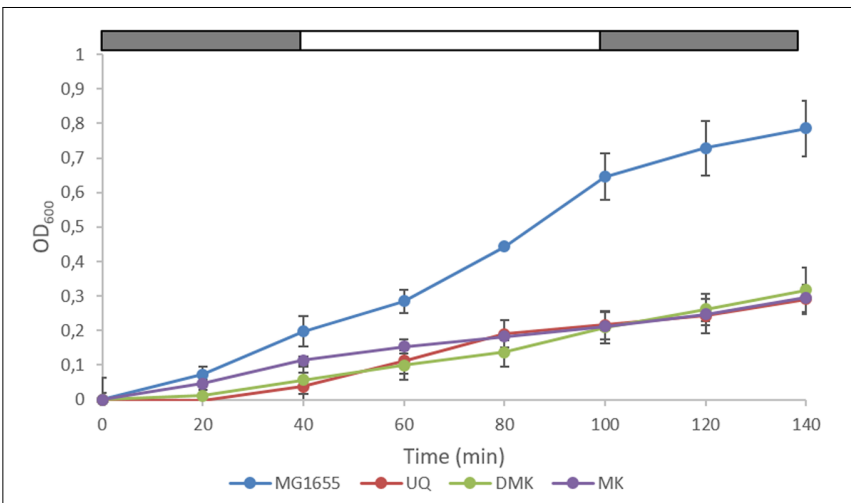

FIGURE 3 | Growth of wild type E. coli and the three single quinone strains in (an)aerobic transition experiments. The last three data points correspond to anaerobic growth, except the MK-only strain for which the last two points are anaerobic. The bar above each panel indicates sparging with $\mathrm{N}_{2}$ (gray) or air (white). Data represent the average of three biologically independent replicates, error bars indicate the standard deviation.

to ArcB's preference for ubi- rather than menaquinols, and the lower midpoint potential of DMK compared to UQ, which may decrease the rate of electron transfer from $\mathrm{DMKH}_{2}$ to $\mathrm{ArcB}$. A similar observation was made in the MK-only strain, where very little $\mathrm{ArcA} \sim \mathrm{P}$ was observed in our anaerobic/aerobic shift assay (Figure 4D).

The MK-only strain showed the lowest level of ArcA $\sim$, but the anaerobic/aerobic/anaerobic trend is clearly visible. These results demonstrate that all three quinone species are able to control the activity or ArcB.

\section{The Redox State/Potential of the (Ubi)quinone Pool}

The quinone pool redox state is considered to be the main regulatory input signal for ArcB-kinase activity, by regulating its switching between kinase and phosphatase activity. In order to link the Q-pool redox state with the ArcA phosphorylation state directly, the Q-pool redox state was measured using an HPLC setup as described before (Bekker et al., 2007; Sharma et al., 2013). Reduced UQ and DMK appeared to be quite stable in hexanol at room temperature for the time period necessary to extract and isolate these quinones. However, $\mathrm{MKH}_{2}$ (auto)oxidized very rapidly. The data presented here on the MK redox state (Supplementary Figure S2) can therefore not be considered to represent the in vivo state.

In the wild type organism, the $\mathrm{UQ} / \mathrm{UQH}_{2}$ ratio seems to follow the anaerobic/aerobic shift reasonably well, being more reduced under anaerobic conditions and becoming oxidized when oxygen is present (Supplementary Figure S2A). Both DMK and $\mathrm{MK}$ are more reduced under initial anaerobic conditions, but as these pools are more rapidly oxidized and less rapidly reduced, no clear peaks are seen with the later samples (e.g., Supplementary Figure S2B). Strikingly, under aerobic conditions, the level of MK dropped below the detection limit in some samples. As soon as anaerobic conditions returned, MK levels rose again. 

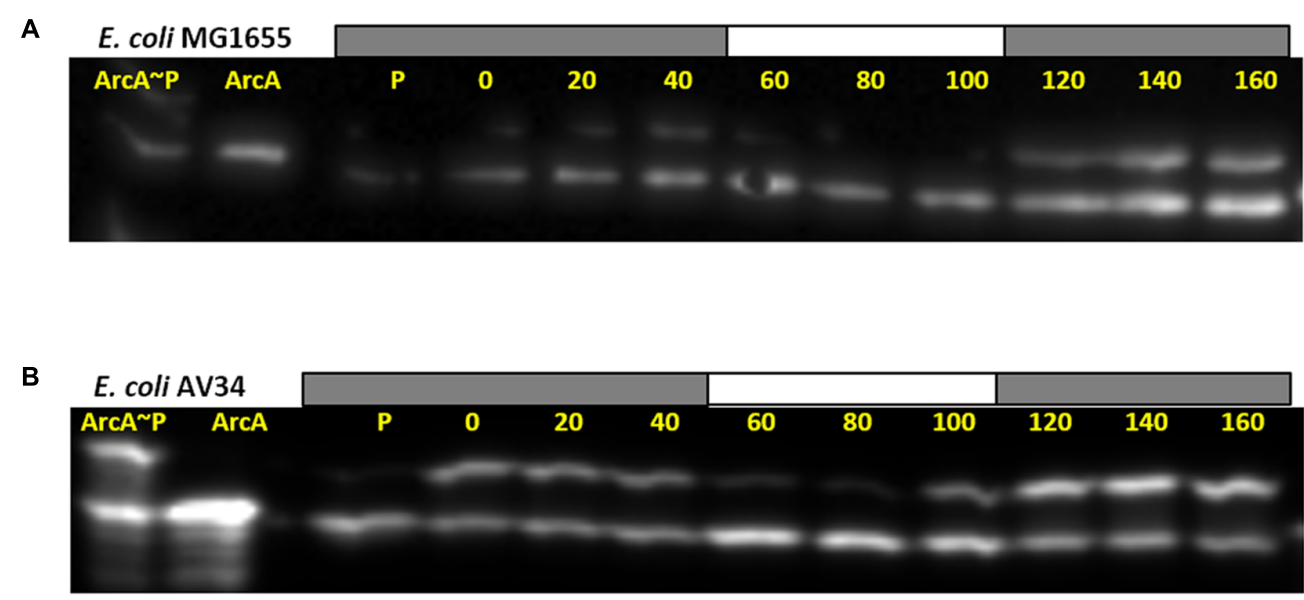

C

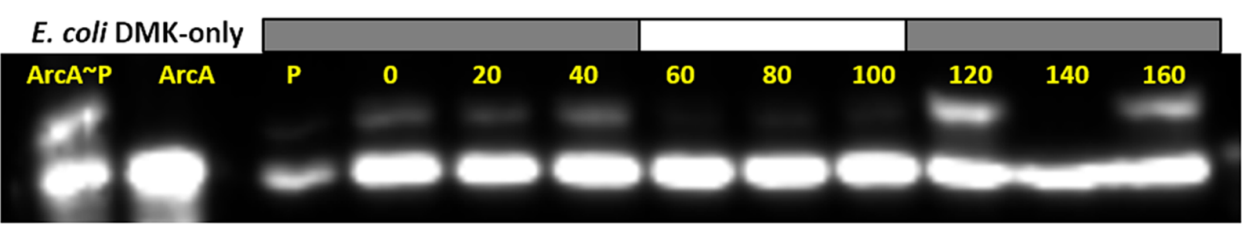

D
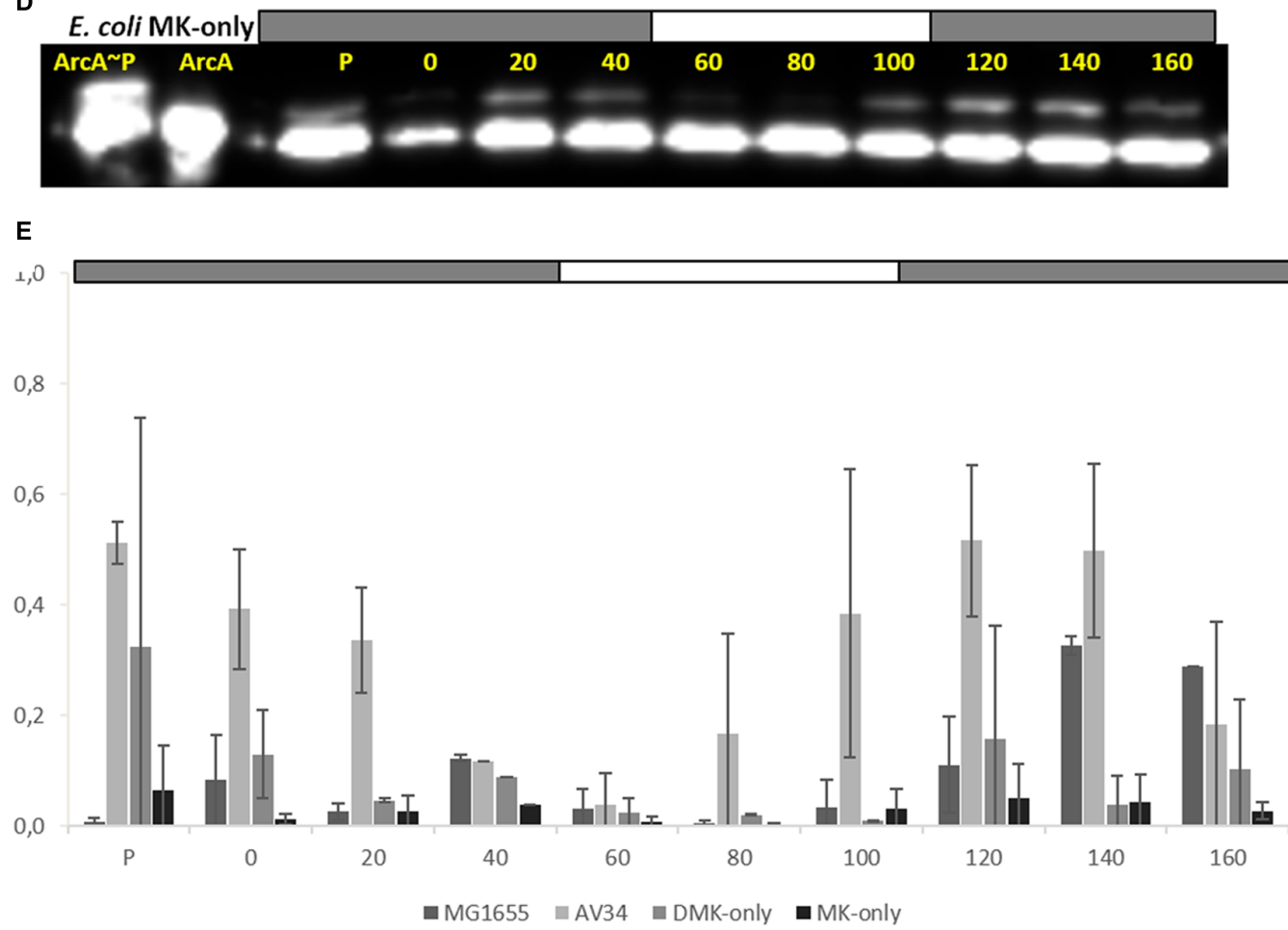

FIGURE 4 | All quinones of E. coli can both reduce (activate) and oxidize (inactivate) ArcAB/A. ArcA P Phos-Tag Western Blot of $E$. coli strain used in this study. The lower band represents ArcA while the upper band represents ArcA P, which is slowed down by the Phos-Tag gel during electorphoresis. The first two lanes are reserved for the ArcA standard, and in-vitro phosphorylated ArcA, respectively. P indicates the pre-culture and the other lanes the sampling time (min). The bar on top of (A-D) indicates $\mathrm{N}_{2}$ (gray) or air (white) sparging. (A) E. coli MG1655, (B) E. coli AV34 (UQ-only), (C) E. coli DMK-only, (D) E. coli MK-only, (E) Graph of the phosphorylation level of ArcA in the different strains used in this study. The bar graphs shows the results of densitometric quantitation of the ArcA P/ArcA ratio. Data represent the average of three biologically independent replicates, error bars indicate the standard deviation. 


\section{Effect of the Quinone Composition on the Pattern of Fermentation Products Formed in the Three Single- Quinone Mutants during (An)aerobic Transitions}

Because metabolic end-products reflect the cell's physiology, the remaining extracellular glucose and several additional exometabolites were analyzed quantitatively with HPLC. As the phosphorylation state of ArcA modulates the expression of several of the respiratory chain components and TCA-cycle enzymes, the degree of activation (i.e., phosphorylation state) of this response regulator might also be inferred from the composition of these metabolic end products.

During anaerobic growth (and also in the pre-culture; data not shown), considerably more formic acid is formed by all three single quinone strains, as compared to the wild type organism (Supplementary Figure S3). This formic acid is rapidly consumed in both MG1655 and AV34 (the UQ only strain) under aerobic conditions. When these strains are switched back to anaerobiosis, formic acid production only slowly resumes. The two menaquinone containing strains, under aerobic conditions, do not appear to catabolize the formic acid produced.

Besides this, MG1655 and AV34 mainly secrete acetate as a result of overflow metabolism (Supplementary Figures S3A,B). Strain AV34 (UQ-only) secretes predominantly acetate under glucose-excess conditions. Strikingly in the two naphtoquinoneonly producing strains, i.e., DMK-only, but especially the MKonly strain, fermentation is mostly directed toward lactate production, thus preventing $\mathrm{NAD}^{+}$depletion.

\section{DISCUSSION}

\section{Construction of the Single-Naphtoquinone Containing Strains of $E$. coli}

We set out to investigate the signals inducing transitions of the activity of ArcB, between its kinase- and phosphatase function. Rather than modulating the concentration of all six quinone species in wild type strains (see Figure 2A) we choose to construct mutants that contain the oxidized and reduced form of a single quinone only. From previous work both a UQ only (AV34) and a 'DMK only' strain (AV36) were available (Sharma et al., 2013). The latter, however, was constructed in such a way that there was considerable risk that a redoxactive intermediate, C1-demethyl-C6-demethoxy-Q $\mathrm{Q}_{8}$ (DDMQ), with a midpoint potential likely more positive than UQ) would accumulate, which then also might become involved in the (de)activation of ArcB. We therefore constructed an improved version of the DMK only strain and a strain containing exclusively $\mathrm{MK}$ as its quinone.

For construction of this latter strain it is relevant to know that, in contrast to E. coli, B. subtilis uses only $\mathrm{MK}$ in its respiratory chain, and that this organism also possesses a cytochrome $c$ oxidase branch in addition to its quinone oxidase branch (Poole and Cook, 2000; Winstedt and von Wachenfeldt, 2000) in its electron transfer chain. Intriguingly, B. subtilis has a stronger preference for aerobic conditions than E. coli does (Nakano and Zuber, 1998). Despite the fact that the route of synthesis of MK is similar in both organisms, no DMK has been observed to be present in B. subtilis, nor is this naphtoquinone assumed to have a biological function in this Gram-positive organism (Kröger and Dadák, 1969; Taber et al., 1981). This prompted us to try the B. subtilis homolog of $\mathrm{UbiE}, \mathrm{MenH}_{\mathrm{Bsu}}$, to convert $\mathrm{DMK}$ to $\mathrm{MK}$ in $E$. coli (Lee et al., 1997). This resulted indeed in very efficient conversion of DMK, which fully depleted the DMK-pool of E. coli and allowed the construction of an MK-only E. coli strain, as was shown by analysis of its quinone complement (Figure 2B). The latter figure also confirms the phenotype of the DMK only strain.

The observation that this improved DMK only strain (i.e., a strain in which DDMQ no longer can accumulate), grows reasonably well also aerobically (see Figure 3 and JWAvB and $\mathrm{KJH}$, unpublished observations) is also relevant because of "the classic view associating ubiquinone to aerobic growth and menaquinone to anaerobic growth" (Aussel et al., 2014): Presumably at least one of the three terminal oxidases of $E$. coli has significant affinity for demethyl-menaquinol. Whether or not the same holds for menaquinol cannot be concluded because of the high frequency with which suppressor mutants appear (see Construction of Three 'Single Quinone' E. coli Strains) under the conditions tested. This mutation frequency might decrease micro-aerobically, as it is possible that reactive oxygen species (ROS) are formed under aerobic conditions that can cause DNA damage (Iuchi and Weiner, 1996).

\section{Quinone Specificity of Signal Input into the Two-Component Sensor ArcB}

It is now generally accepted that ubiquinone inhibits the kinase activity of $\mathrm{ArcB}$ and therefore activates its phosphatase function (10). However, whether the other quinones of E. coli are capable of doing so has been debated. Based on the relation between the rate of oxygen consumption and $\mathrm{ArcB} / \mathrm{A}$ activity we proposed in 2010 that besides ubiquinone, also a naphtoquinone must be able to switch ArcB into its phosphatase function (Sharma et al., 2012).

Although, meanwhile we have provided more evidence supporting our interpretation (Sharma et al., 2013), Georgellis et al. have published an updated model for the regulation of ArcB activity by the quinone pools of $E$. coli, based on an in vivo determination of the redox midpoint potential of the redox-sensitive active site cysteine(s) residue of ArcB (Alvarez et al., 2013). They propose that ubiquinone (with a midpoint potential of $+100 \mathrm{mV}$ ) can only inactivate $\mathrm{ArcB}$, because ubiquinol would not be able to transfer electrons to the cysteines of ArcB. This latter conclusion was presumably also proposed based on earlier work in which it was shown that $\mathrm{UQ}_{0} \mathrm{H}_{2}$ and menadione in vitro cannot activate $\mathrm{ArcB}$ (for review see Malpica et al., 2006). According to their interpretation only the two naphtoquinols can activate $\operatorname{ArcB}$ (13). However, through the use of a so-called single quinone mutants we had shown that both ubiquinol and presumably also menaquinol can activate ArcB in E. coli (Bekker et al., 2010; Sharma et al., 2012). 
We here show that all three types of $E$. coli cells with a linear respiratory chain at the quinone level are capable of regulating (i.e., activating and deactivating) the activity $\mathrm{ArcB}$, that is, in all three single-quinone strains, the $\operatorname{ArcA} / \mathrm{ArcA} \sim \mathrm{P}$ ratio responds to modulation of the redox state of the respective quinone pool through variation of oxygen availability. For both naphtoquinone-containing strains this is new evidence; for the DMK only strain because we can now exclude that the biosynthetic intermediate C1-demethyl-C6-demethoxy- $\mathrm{Q}_{8}$ might have been responsible for modulation of $\mathrm{ArcB}$ activity in this strain instead (see also above).

In our anaerobic/aerobic transition experiments, low levels of ArcA $\sim P$ were found in the (D)MK-only strains. Presumably, residual amounts of oxygen and/or DMSO are still being reduced, which helps to keep the respective quinone pool in a partially oxidized state. Here, it is also relevant to note that SixA may inhibit the kinase activity of ArcB when alternative electron acceptors are available (Matsubara and Mizuno, 2000). Nevertheless, also long-term anaerobic incubation showed clearly that the $\mathrm{ArcB} / \mathrm{A}$ system is functional and ArcA can be phosphorylated in vivo in these strains (our unpublished results). These results re-enforce the interpretations made in our earlier studies $(6,8)$. In these interpretations the first-order assumption would be that all three quinols activate $\mathrm{ArcB}$ with the same rate. At this stage it seems that this assumption does not fully hold. But then again of course rates of $\mathrm{ArcB}$ activation are not only determined by quinol specificity, but also by their concentration which are partly determined via the redox potential of the respective pools.

\section{Effect of Single Quinone Mutations on the Fermentation Products Produced during (An)aerobic Transitions in Batch Cultures of E. coli}

Several notable effects can be observed in the pattern of formation of fermentation products in the wild type and in the three single quinone mutants in batch cultures undergoing (an)aerobiosis transitions (Supplementary Figure S3). Glucose is indeed much faster consumed in the wild type than in the three mutants, consistent with the growth rate of the four strains (Figure 3), be it that a significant part of it is converted into acetate and ethanol via overflow metabolism (Liao and Farmer, 1997; Picon et al., 2005). Succinate production is considerably lowered in the two naphtoquinone-only mutants, as compared to the wild type strain, consistent with the fact that the fumarate reductase enzyme is specific for menaquinone (Wallace and Young, 1977). However, this lowered succinate production is also observed in the UQ only mutant (Supplementary Figure S3B). Formate does not appear to be degraded by the DMK and MK-only strains, in agreement with earlier reports (Wallace and Young, 1977). The most striking difference between the four strains is the high rate of lactate formation, which goes at the cost of formation of the other fermentation products, in the two mutants that cannot make ubiquinone. This might confirm that the respiratory lactate dehydrogenase
(LdhA) is highly specific for ubiquinone as the electron acceptor, as it would produce D-lactate (Futai, 1973; Wallace and Young, 1977; Matsushita and Kaback, 1986). However, the methylglyoxal pathway could produce significant amounts of lactate $(\mathrm{L}+\mathrm{D})$, which would bypass central metabolism completely. To what extent this shunt is used is beyond the scope of this investigation. There has been speculation in the literature that specific fermentation products, e.g., D-lactate, might also modulate the activity of $\mathrm{ArcB}$ (Georgellis et al., 1999; Rodriguez et al., 2004), without, however, proposing a mechanism for this. We think that the current results do not provide incentives to try and propose such a mechanism (Malpica et al., 2006).

\section{Measurement of the Redox State of the Pools of the Naphtoquinones}

In experiments with Bacillus megaterium, the menaquinone pool has been observed to be in a highly reduced state (up to $85 \%$ reduced) when cells were grown anaerobically, while in the same cells under aerobic conditions this pool was found to be only $10 \%$ reduced (Kröger et al., 1971). However, to obtain these results an approach is required that uses specialized equipment; and this approach is only suitable for species with a single type of quinone. DMK too has been assessed in a similar fashion, in Hemophilus parainfluenzae (White, 1965). These levels for MK and DMK are not very different from what has been observed in E. coli for UQ (Bekker et al., 2007). It would be very interesting to find out whether DMK and $\mathrm{MK}$ can be reduced to a similarly high level in E. coli, but such experiments are technically very challenging. It is relevant to note that with a similar procedure as the one used here, plus extra precautions for very rapid analysis, it was possible to analyze the in vivo redox state of plastoquinone in Synechocystis sp. PCC6803 (Schuurmans et al., 2014). We do anticipate, however, that the data on the in vivo amount of reduced naphtoquinones reported in this contribution represent a gross underestimate due to the rapid autooxidation of these quinones.

\section{Outlook}

To understand the modulation of the activity of ArcB in even more detail multi-omics analyses will be necessary, similar to the ones recently published for glucose repression (Borirak et al., 2015). That approach provides a more detailed picture of the metabolic consequences of $\mathrm{ArcB}$ (de)activation, and also an independent and complementary way to assay this via transcript profiling (e.g., Wareham et al., 2016). In such future experiments it will be an asset to be able to modulate the size of the proton motive force independently and orthogonally, e.g., with a light-dependent proton pump like proteorhodopsin, because of the recent report that that will allow an independent modulation of the rate of formation of ROS (see Na et al., 2015). Such an approach would then also allow for an independent test whether the size of the proton motive force and ROS formation can or cannot directly modulate the activity of ArcB (Bogachev et al., 1995; Iuchi and Weiner, 1996; Malpica et al., 2004). 


\section{AUTHOR CONTRIBUTIONS}

$\mathrm{JvB}$ and $\mathrm{KH}$ jointly designed the study, JvB carried out the experiments, and $\mathrm{KH}$ and JvB jointly interpreted the results and wrote the paper.

\section{FUNDING}

This work was supported by the SysMo-SUMO2 project, a European Transnational Funding and Research Initiative.

\section{REFERENCES}

Alexeeva, S., Hellingwerf, K. J., and Teixeira de Mattos, M. J. (2002). Quantitative assessment of oxygen availability: perceived aerobiosis and its effect on flux distribution in the respiratory chain of Escherichia coli. J. Bacteriol. 184, $1402-$ 1406. doi: 10.1128/JB.184.5.1402

Alvarez, A. F., Rodriguez, C., and Georgellis, D. (2013). Ubiquinone and menaquinone electron carriers represent the yin and yang in the redox regulation of the ArcB sensor kinase. J. Bacteriol. 195, 3054-3061. doi: 10.1128/JB.00406-13

Aussel, L., Pierrel, F., Loiseau, L., Lombard, M., Fontecave, M., and Barras, F. (2014). Biosynthesis and physiology of coenzyme Q in bacteria. Biochim. Biophys. Acta 1837, 1004-1011. doi: 10.1016/j.bbabio.2014. 01.015

Bekker, M., Alexeeva, S., Laan, W., Sawers, G., Teixeira de Mattos, J., and Hellingwerf, K. (2010). The ArcBA two-component system of Escherichia coli is regulated by the redox state of both the ubiquinone and the menaquinone pool. J. Bacteriol. 192, 746-754. doi: 10.1128/JB.01156-09

Bekker, M., De Vries, S., Ter Beek, A., Hellingwerf, K. J., and Teixeira De Mattos, M. J. (2009). Respiration of Escherichia coli can be fully uncoupled via the nonelectrogenic terminal cytochrome bd-II oxidase. J. Bacteriol. 191, 55105517. doi: 10.1128/JB.00562-09

Bekker, M., Kramer, G., Hartog, A. F., Wagner, M. J., de Koster, C. G., Hellingwerf, K. J., et al. (2007). Changes in the redox state and composition of the quinone pool of Escherichia coli during aerobic batch-culture growth. Microbiology 153, 1974-1980. doi: 10.1099/mic.0.2007/006098-0

Berg, J. M., Tymoczko, J. L., and Stryer, L. (2002). Biochemistry, 5th Edn. New York, NY: W H Freeman.

Bogachev, A. V., Murtazine, R. A., Shestopalov, A. I., and Skulachev, V. P. (1995). Induction of the Escherichia coli cytochrome d by low delta $\mathrm{mu} \mathrm{H}^{+}$ and by sodium ions. Eur. J. Biochem. 232, 304-308. doi: 10.1111/j.14321033.1995.tb20812.x

Borirak, O., Rolfe, M. D., de Koning, L. J., Hoefsloot, H. C. J., Bekker, M., Dekker, H. L., et al. (2015). Time-series analysis of the transcriptome and proteome of Escherichia coli upon glucose repression. Biochim. Biophys. Acta 1854, 1269-1279. doi: 10.1016/j.bbapap.2015. 05.017

Evans, C. G. T., Herbert, D., and Tempest, D. W. (1970). "The continuous cultivation of micro-organisms," in Methods in Microbiology, eds J. R. Norris and D. W. Ribbons (London: Academic Press), 277-327. doi: 10.1016/S05809517(08)70227-7

Futai, M. (1973). Membrane D-lactate dehydrogenase from Escherichia coli. purification and properties. Biochemistry 12, 2468-2474. doi: 10.1021/bi00737a016

Georgellis, D., Kwon, O., and Lin, E. C. (1999). Amplification of signaling activity of the arc two-component system of Escherichia coli by anaerobic metabolites. An in vitro study with different protein modules. J. Biol. Chem. 274, 35950-35954. doi: $10.1074 /$ jbc. 274.50 .35950

Georgellis, D., Kwon, O., and Lin, E. C. (2001). Quinones as the redox signal for the arc two-component system of bacteria. Science 292, 2314-2316. doi: $10.1126 /$ science. 1059361

\section{ACKNOWLEDGMENT}

Jos Arents is acknowledged for his assistance with sampling and the Western blots. $\mathrm{KJH}$ would like to dedicate this contribution to the memory of his mentor WN Konings who passed away on July 5th, 2015.

\section{SUPPLEMENTARY MATERIAL}

The Supplementary Material for this article can be found online at: http://journal.frontiersin.org/article/10.3389/fmicb. 2016.01339

Guérout-Fleury, A. M., Frandsen, N., and Stragier, P. (1996). Plasmids for ectopic integration in Bacillus subtilis. Gene 180, 57-61. doi: 10.1016/S03781119(96)00404-0

Hellingwerf, K. J., Bolscher, J. G., and Konings, W. N. (1981). The electrochemical proton gradient generated by the fumarate-reductase system in Escherichia coli and its bioenergetic implications. Eur. J. Biochem. 113, 369-374. doi: 10.1111/j.1432-1033.1981.tb05075.x

Hellingwerf, K. J., and Konings, W. N. (1985). The energy flow in bacteria: the main free energy intermediates and their regulatory role. Adv. Microb. Physiol. 26, 125-154. doi: 10.1016/S0065-2911(08)60396-3

Holländer, R. (1976). Correlation of the function of demethylmenaquinone in bacterial electron transport with its redox potential. FEBS Lett. 72, 98-100. doi: 10.1016/0014-5793(76)80821-6

Iuchi, S., and Weiner, L. (1996). Cellular and molecular physiology of Escherichia coli in the adaptation to aerobic environments. J. Biochem. 120, 1055-1063. doi: 10.1093/oxfordjournals.jbchem.a021519

Keseler, I. M., Mackie, A., Peralta-Gil, M., Santos-Zavaleta, A., Gama-Castro, S., Bonavides-Martínez, C., et al. (2013). EcoCyc: fusing model organism databases with systems biology. Nucleic Acids Res. 41, D605-D612. doi: 10.1093/nar/gks1027

Kröger, A., and Dadák, V. (1969). On the role of quinones in bacterial electron transport. The respiratory system of Bacillus megaterium. Eur. J. Biochem. 11, 328-340. doi: 10.1111/j.1432-1033.1969.tb00776.x

Kröger, A., Dadák, V., Klingenberg, M., and Diemer, F. (1971). On the role of quinones in bacterial electron transport. Differential roles of ubiquinone and menaquinone in Proteus rettgeri. Eur. J. Biochem. 21, 322-333. doi: 10.1111/j.1432-1033.1971.tb01472.x

Lee, D. J., Bingle, L. E. H., Heurlier, K., Pallen, M. J., Penn, C. W., Busby, S. J. W., et al. (2009). Gene doctoring: a method for recombineering in laboratory and pathogenic Escherichia coli strains. BMC Microbiol. 9:252. doi: 10.1186/14712180-9-252

Lee, P. T., Hsu, A. Y., Ha, H. T., and Clarke, C. F. (1997). A C-methyltransferase involved in both ubiquinone and menaquinone biosynthesis:isolation and identification of the Escherichia coli ubiE gene. J. Bacteriol. 179, 1748-1754.

Liao, J. C., and Farmer, W. R. (1997). Reduction of aerobic acetate production by Escherichia coli. Appl. Environ. Microbiol. 63, 3205-3210.

Madigan, M. T., and Martinko, J. M. (2014). Brock Biology of Microorganisms, 14th Edn. Upper Saddle River, NJ: Pearson Education.

Malpica, R., Franco, B., Rodriguez, C., Kwon, O., and Georgellis, D. (2004). Identification of a quinone-sensitive redox switch in the $\mathrm{ArcB}$ sensor kinase. Proc. Natl. Acad. Sci. U.S.A. 101, 13318-13323. doi: 10.1073/pnas.0403064101

Malpica, R., Sandoval, G. R. P., Rodríguez, C., Franco, B., and Georgellis, D. (2006). Signaling by the arc two-component system provides a link between the redox state of the quinone pool and gene expression. Antioxid. Redox Signal. 8, 781-795. doi: 10.1089/ars.2006.8.781

Matsubara, M., and Mizuno, T. (2000). The SixA phospho-histidine phosphatase modulates the ArcB phosphorelay signal transduction in Escherichia coli. FEBS Lett. 470, 118-124. doi: 10.1016/S0014-5793(00)01303-X

Matsushita, K., and Kaback, H. R. (1986). D-lactate oxidation and generation of the proton electrochemical gradient in membrane vesicles from Escherichia coli GR19N and in proteoliposomes reconstituted with purified D-lactate 
dehydrogenase and cytochrome o oxidase. Biochemistry 25, 2321-2327. doi: 10.1021/bi00357a004

Meganathan, R. (2001a). Biosynthesis of menaquinone (vitamin K2) and ubiquinone (coenzyme Q): a perspective on enzymatic mechanisms. Vitam. Horm. 61, 173-218. doi: 10.1016/S0083-6729(01)61006-9

Meganathan, R. (2001b). Ubiquinone biosynthesis in microorganisms. FEMS Microbiol. Lett. 203, 131-139. doi: 10.1111/j.1574-6968.2001.tb10831.x

Messner, K. R., and Imlay, J. A. (1999). The identification of primary sites of superoxide and hydrogen peroxide formation in the aerobic respiratory chain and sulfite reductase complex of Escherichia coli. J. Biol. Chem. 274, 1011910128. doi: 10.1074/jbc.274.15.10119

Na, Y. A., Lee, J. Y., Bang, W. J., Lee, H. J., Choi, S. I., Kwon, S. K., et al. (2015). Growth retardation of Escherichia coli by artificial increase of intracellular ATP. J. Ind. Microbiol. Biotechnol. 42, 915-924. doi: 10.1007/s10295-015-1609-6

Nakano, M. M., and Zuber, P. (1998). Anaerobic growth of a "strict aerobe" (Bacillus subtilis). Annu. Rev. Microbiol. 52, 165-190. doi: 10.1146/annurev.micro.52.1.165

Nicholls, D., and Ferguson, S. (2013). Bioenergetics, 4th Edn. Philadelphia, PA: Elsevier Science.

Picon, A., Teixeira de Mattos, M. J., and Postma, P. W. (2005). Reducing the glucose uptake rate in Escherichia coli affects growth rate but not protein production. Biotechnol. Bioeng. 90, 191-200. doi: 10.1002/bit.20387

Poole, R. K., and Cook, G. M. (2000). Redundancy of aerobic respiratory chains in bacteria? Routes, reasons and regulation. Adv. Microb. Physiol. 43, 165-224. doi: 10.1016/S0065-2911(00)43005-5

Rodriguez, C., Kwon, O., and Georgellis, D. (2004). Effect of D-Lactate on the physiological activity of the ArcB sensor kinase in Escherichia coli. J. Bacteriol. 186, 2085-2090. doi: 10.1128/JB.186.7.2085

Rolfe, M. D., Ter Beek, A., Graham, A. I., Trotter, E. W., Asif, H. M. S., Sanguinetti, G., et al. (2011). Transcript profiling and inference of Escherichia coli K-12 ArcA activity across the range of physiologically relevant oxygen concentrations. J. Biol. Chem. 286, 10147-10154. doi: 10.1074/jbc.M110.211144

Salmon, K. A., Hung, S., Steffen, N. R., Krupp, R., Baldi, P., Hatfield, G. W., et al. (2005). Global gene expression profiling in Escherichia coli K12: effects of oxygen availability and ArcA. J. Biol. Chem. 280, 15084-15096. doi: 10.1074/jbc.M414030200

Schuurmans, R. M., Schuurmans, J. M., Bekker, M., Kromkamp, J. C., Matthijs, H. C. P., and Hellingwerf, K. J. (2014). The redox potential of the plastoquinone pool of the cyanobacterium Synechocystis species strain PCC 6803 is under strict homeostatic control. Plant Physiol. 165, 463-475. doi: 10.1104/pp.114.237313

Sharma, P., Stagge, S., Bekker, M., Bettenbrock, K., and Hellingwerf, K. J. (2013). Kinase activity of ArcB from Escherichia coli is subject to regulation by both Ubiquinone and Demethylmenaquinone. PLoS ONE 8:e75412. doi: 10.1371/journal.pone.0075412

Sharma, P., Teixeira de Mattos, M. J., Hellingwerf, K. J., and Bekker, M. (2012). On the function of the various quinone species in Escherichia coli. FEBS J. 279, 3364-3373. doi: $10.1111 / j .1742-4658.2012 .08608 . x$

Steinsiek, S., Stagge, S., and Bettenbrock, K. (2014). Analysis of Escherichia coli mutants with a linear respiratory chain. PLOS ONE 9:e87307. doi: 10.1371/journal.pone.0087307

Taber, H. W., Dellers, E. A., and Lombardo, L. R. (1981). Menaquinone biosynthesis in Bacillus subtilis: isolation of men mutants and evidence for clustering of men genes. J. Bacteriol. 1, 321-327.
Tran, Q. H., and Unden, G. (1998). Changes in the proton potential and the cellular energetics of Escherichia coli during growth by aerobic and anaerobic respiration or by fermentation. Eur. J. Biochem. 251, 538-543. doi: 10.1046/j.1432-1327.1998.2510538.x

Unden, G., and Bongaerts, J. (1997). Alternative respiratory pathways of Escherichia coli: energetics and transcriptional regulation in response to electron acceptors. Biochim. Biophys. Acta 1320, 217-234. doi: 10.1016/S00052728(97)00034-0

Van Der Plas, J., Hellingwerf, K. J., Seijen, H. G., Guest, J. R., Weiner, J. H., and Konings, W. N. (1983). Identification and localizationof enzymes of the fumarate reductase and nitrate respirationsystems of Escherichia coli by crossed immunoelectrophoresis. J. Bacteriol. 153, 1027-1037.

van Schie, B. J., Hellingwerf, K. J., van Dijken, J. P., Elferink, M. G., van Dijl, J. M., Kuenen, J. G., et al. (1985). Energy transduction by electron transfer via a pyrrolo-quinoline quinone-dependent glucose dehydrogenase in Escherichia coli, Pseudomonas aeruginosa, and Acinetobacter calcoaceticus (var. lwoffi). J. Bacteriol. 163, 493-499.

Visser, R. G., Hellingwerf, K. J., and Konings, W. N. (1984). The protein composition of the cytoplasmic membrane of aerobically and anaerobically grown Escherichia coli. J. Bioenerg. Biomembr. 16, 295-307. doi: $10.1007 / \mathrm{BF} 00744282$

Wallace, B., and Young, I. (1977). Role of quinones in electron transport to oxygen and nitrate in Escherichia coli. Studies with a ubiA- menA- double quinone mutant. Biochim. Biophys. Acta 461, 84-100. doi: 10.1016/0005-2728(77) 90071-8

Wang, X., Kim, Y., Ma, Q., Hong, S. H., Pokusaeva, K., Sturino, J. M., et al. (2010). Cryptic prophages help bacteria cope with adverse environments. Nat. Commun. 1, 147. doi: 10.1038/ncomms1146

Wareham, L. K., Begg, R., Jesse, H. E., Van Beilen, J. W. A., Ali, S., Svistunenko, D., et al. (2016). Carbon monoxide gas is not inert, but global, in its consequences for bacterial gene expression, iron acquisition, and antibiotic resistance. Antioxid. Redox Signal. 24, 1013-1028. doi: 10.1089/ars.2015.6501

White, D. C. (1965). The funtions of 2-demethyl vitamin K2 in the electron transport sytem of Haemophilus parainfluenzae. J. Biol. Chem. 240, 1387-1394.

Winstedt, L., and von Wachenfeldt, C. (2000). Terminal oxidases of Bacillus subtilis strain 168: one quinol oxidase, cytochrome aa(3) or cytochrome bd, is required for aerobic growth. J. Bacteriol. 182, 6557-6564. doi: 10.1128/JB.182.23.65576564.2000

Wissenbach, U., Kröger, A., and Unden, G. (1990). The specific functions of menaquinone and demethylmenaquinone in anaerobic respiration with fumarate, dimethylsulfoxide, trimethylamine $\mathrm{N}$-oxide and nitrate by Escherichia coli. Arch. Microbiol. 154, 60-66. doi: 10.1007/BF00249179

Conflict of Interest Statement: The authors declare that the research was conducted in the absence of any commercial or financial relationships that could be construed as a potential conflict of interest.

Copyright (C) 2016 van Beilen and Hellingwerf. This is an open-access article distributed under the terms of the Creative Commons Attribution License (CC BY). The use, distribution or reproduction in other forums is permitted, provided the original author(s) or licensor are credited and that the original publication in this journal is cited, in accordance with accepted academic practice. No use, distribution or reproduction is permitted which does not comply with these terms. 